PROCEEDINGS OF THE

AMERICAN MATHEMATICAL SOCIETY

Volume 132, Number 11, Pages 3449-3456

S 0002-9939(04)07505-7

Article electronically published on April 9, 2004

\title{
A CRITERION FOR SATELLITE 1-GENUS 1-BRIDGE KNOTS
}

\author{
HIROSHI GODA, CHUICHIRO HAYASHI, AND HYUN-JONG SONG
}

(Communicated by Ronald A. Fintushel)

\begin{abstract}
Let $K$ be a knot in a closed orientable irreducible 3-manifold $M$. Suppose $M$ admits a genus 1 Heegaard splitting and we denote by $H$ the splitting torus. We say $H$ is a 1-genus 1-bridge splitting of $(M, K)$ if $H$ intersects $K$ transversely in two points, and divides $(M, K)$ into two pairs of a solid torus and a boundary parallel arc in it. It is known that a 1-genus 1-bridge splitting of a satellite knot admits a satellite diagram disjoint from an essential loop on the splitting torus. If $M=S^{3}$ and the slope of the loop is longitudinal in one of the solid tori, then $K$ is obtained by twisting a component of a 2-bridge link along the other component. We give a criterion for determining whether a given 1-genus 1-bridge splitting of a knot admits a satellite diagram of a given slope or not. As an application, we show there exist counter examples for a conjecture of Ait Nouh and Yasuhara.
\end{abstract}

\section{INTRODUCTION}

Let $M$ be a closed orientable irreducible 3-manifold, and $K$ a knot in $M$. We say that $K$ is a 1-genus 1-bridge knot if $(M, K)$ has a 1-genus 1-bridge splitting $H$, that is, there is a Heegaard splitting torus $H$ of $M$ such that $H$ intersects $K$ transversely in two points and $K$ intersects each of the solid tori bounded by $H$ in a trivial arc. (Here, an arc $t$ embedded in a solid torus $V$ with $t \cap \partial V=\partial t$ is called trivial if it is boundary parallel, that is, there is a disc $C$ in $V$ such that $t \subset \partial C$ and $C \cap \partial V=\operatorname{cl}(\partial C-t)$. We call such a disc $C$ a cancelling disc of $t$.) The class of 1-genus 1-bridge knots contains all torus knots and 2-bridge knots.

Let $(M, K)=\left(V_{1}, t_{1}\right) \cup_{H}\left(V_{2}, t_{2}\right)$ be a 1-genus 1-bridge splitting, and $C_{i}$ a cancelling disc of $t_{i}$ in $V_{i}$ for $i=1$ and 2. Set $s_{i}=\partial C_{i} \cap H$. Then the overstrand $s_{1}$ and the understrand $s_{2}$ together give a 1-genus 1-bridge diagram of the splitting. It is a satellite diagram if there is an essential loop $\ell$ in $H$ with $\ell \cap\left(s_{1} \cup s_{2}\right)=\emptyset$. We call the isotopy class of such a loop $\ell$ in $H$ (rather than $H-K$ ) a slope of the satellite diagram. A 1-genus 1-bridge splitting admits a satellite diagram if there is such a pair of cancelling discs. See [5], and also [8]. If the slope of a 1-genus

Received by the editors March 17, 2003 and, in revised form, August 11, 2003.

2000 Mathematics Subject Classification. Primary 57M25.

Key words and phrases. 2-bridge link, twisting operation, 1-genus 1-bridge knot, satellite diagram.

This work was supported by Joint Research Project 'Geometric and Algebraic Aspects of Knot Theory', under the Japan-Korea Basic Scientific Cooperation Program by KOSEF and JSPS. The authors would like to thank Professor Hitoshi Murakami for giving us this opportunity.

(C)2004 American Mathematical Society 


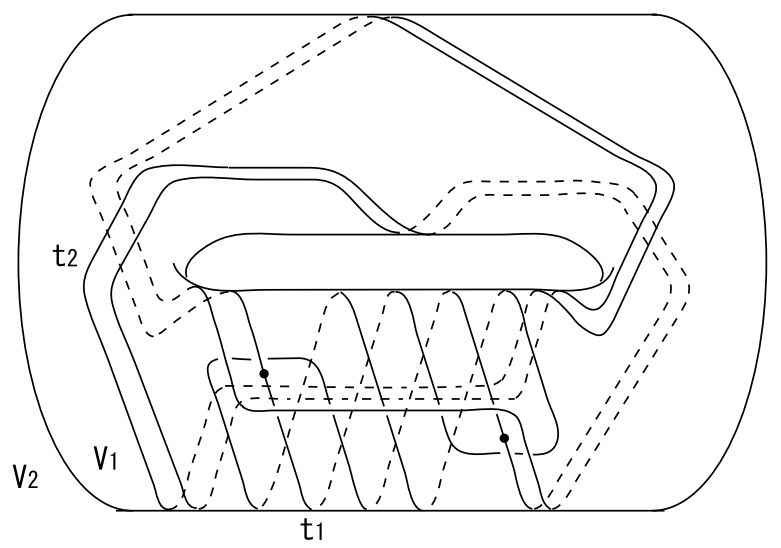

FIGURE 1.

1-bridge satellite diagram is meridional in one of the solid tori $V_{1}$ and $V_{2}$, then the knot $K$ is trivial. When the slope is longitudinal on $\partial V_{i}, K$ can be obtained from a component of a 2-bridge link by Dehn surgery on the other component, as is essentially shown in 8 . (In fact, $K$ has a 1-bridge diagram on the annulus $A=\operatorname{cl}\left(\partial V_{i}-N(\ell)\right)$; that is, shrinking $C_{1}$ and $C_{2}$, we can isotope $K$ to be the union of an overstrand very near to $s_{1}$ and an understrand very close to $s_{2}$. We can take a core of the other solid torus $V_{j}$ to be disjoint from $C_{1}$ and $C_{2}$. We perform a Dehn surgery on the core so that $\partial A$ bounds two meridian $\operatorname{discs} Q$ of the filled solid torus. Thus $K$ is deformed to be in a 1-bridge position with respect to the 2-sphere $A \cup Q$.) When $M=S^{3}$, the Dehn surgery is the same operation as a twisting.

In this paper, we give a criterion for determining whether a given 1-genus 1bridge splitting of a knot has a satellite diagram of a given slope or not. Note that every 1-genus 1-bridge splitting has infinitely many diagrams, since a trivial arc in a solid torus has infinitely many isotopy classes of cancelling discs. (In fact, a trivial $\operatorname{arc} t$ is isotopic in the solid torus $V$ to every arc $\alpha$ in $\partial V$ such that $\partial \alpha=\partial t$ and such that $\alpha$ is disjoint from a meridian disc $D$ of $V$ with $D \cap t=\emptyset$. See Lemma 2.5 in [6].) A 1-genus 1-bridge diagram of a satellite knot is not always satellite even if the overstrand and the understrand intersect each other in a minimal number of points up to isotopy in $H$ fixing their endpoints. See Figure 1 where a cable knot of the trefoil knot is described. In fact, the projection of the $\operatorname{arc} t_{1}$ is isotopic in $V_{1}$ to the straight line connecting the two points $H \cap K$.

However, the Heegaard diagram of a 1-genus 1-bridge splitting is unique up to homeomorphism for the homeomorphism class of the splitting. Here the Heegaard diagram is a pair of isotopy classes of meridian loops $m_{1}$ and $m_{2}$ of $V_{1}$ and $V_{2}$ in the twice punctured torus $H-K$ such that $m_{i}$ bounds a meridian disc disjoint from $t_{i}$ in $V_{i}$. (Note that such a meridian disc is unique up to isotopy of the pair $\left(V_{i}, t_{i}\right)$. See Lemma 2.4 in [6].) We can easily obtain the Heegaard diagram from a 1 -genus 1-bridge diagram. We use Heegaard diagrams in our criterion instead of 1-genus 1-bridge diagrams.

Theorem 1.1. Let $(M, K)=\left(V_{1}, t_{1}\right) \cup_{H}\left(V_{2}, t_{2}\right)$ be a 1-genus 1-bridge splitting, and $D_{i}$ a meridian disc of $V_{i}$ with $D_{i} \cap t_{i}=\emptyset$ for $i=1$ and 2 . Suppose that $\partial D_{1} \cap \partial D_{2}$ 
is minimized up to isotopy in $H-K$. This splitting has a satellite 1-genus 1-bridge diagram of slope $\ell_{0}$ if and only if there is a simple closed curve $\ell$ isotopic to $\ell_{0}$ in $H$ such that $\ell \cup \partial D_{i}$ does not separate the two points $H \cap K$ for $i=1$ and 2 .

Addendum 1.2. (1) In the "only if part", we can take $\ell$ so that $\left|\ell \cap \partial D_{i}\right|=$ $\left|\ell \cdot \partial D_{i}\right|$ for $i=1$ and 2 , where $\ell \cdot \partial D_{i}$ denotes the algebraic intersection number.

(2) The "if part" holds even if $\partial D_{1}$ and $\partial D_{2}$ do not intersect each other in a minimal number of points up to isotopy in $H-K$.

The proof is given in Section 2. We apply this result to torus knots in Section 3 , and obtain the next result.

Corollary 1.3. A 1-genus 1-bridge splitting of a torus knot $T(p, q)$ with $q=p+2$ has a satellite diagram of slope $(1,1)$. In particular, $T(p, p+2)$ is obtained from a component of a 2-bridge link in $S^{3}$ by twisting along the other component.

This corollary gives counterexamples for a conjecture by Ait Nouh and Yasuhara 1], which says if a torus knot $T(p, q)$ is obtained by twisting a trivial knot, then $q=k p \pm 1$ for some integer $k$. The type of the 2-bridge link will be given in [3]. Such a 2-bridge link admits infinitely many exceptional Dehn surgeries, since an $n$-twisting is realized also by a $(-1 / n)$-Dehn surgery, and no Dehn surgery on a torus knot yields a hyperbolic 3-manifold.

In Section 4, applying Theorem 1.1 we show that any 1-genus 1-bridge splitting of the torus knot $T(5,12)$ does not admit a satellite diagram of longitudinal slope of one of the solid tori bounded by the splitting torus. A similar argument works for $T(p, q)$ with $p=4 k+1, q=n p+2$ for $k \geq 1$ and $n \geq 2$.

\section{Proof of Theorem 1.1}

We prove Theorem 1.1 and its addendum in this section.

First we prove the "if part". Since $\ell \cup \partial D_{i}$ does not separate the two points $\partial t_{i}$, there is an $\operatorname{arc} \alpha_{i}$ (in $H$ ) such that $\partial \alpha_{i}=\partial t_{i}$ and $\alpha_{i} \cap\left(\ell \cup \partial D_{i}\right)=\emptyset$. Lemma 2.5 in [6] allows us to take a cancelling $\operatorname{disc} C_{i}$ of $t_{i}$ in $V_{i}$ so that $C_{i} \cap H=\alpha_{i}$. This is because we obtain a ball by cutting $V_{i}$ along $D_{i}$. Thus the $\operatorname{discs} C_{1}$ and $C_{2}$ give a satellite 1-genus 1-bridge diagram disjoint from $\ell$.

Now we prove the "only if part". Suppose that the 1-genus 1-bridge splitting $(M, K)=\left(V_{1}, t_{1}\right) \cup_{H}\left(V_{2}, t_{2}\right)$ has a satellite diagram with slope $\ell$. Then there is a cancelling disc $C_{i}$ of $t_{i}$ in $V_{i}$ such that the $\operatorname{arc} s_{i}=C_{i} \cap H$ is disjoint from $\ell$ for $i=1$ and 2. We can assume that $C_{1}$ and $C_{2}$ are isotoped so that $s_{1}$ and $s_{2}$ intersect each other in a minimal number of points. Let $A$ be the annulus obtained by cutting $H$ along $\ell$. For $i=1$ and 2, we will find a meridian disc $D_{i}$ of $V_{i}$ with $D_{i} \cap t_{i}=\emptyset$ and with the following properties:

(1) $\partial D_{i}$ either intersects $A$ in essential arcs, or is entirely contained in $\operatorname{int} A$.

(2) $\ell \cup \partial D_{i}$ is disjoint from the arc $s_{i}$ for $i=1$ and 2 .

(3) $\partial D_{i} \cup s_{j}$ has no bigon in $H-K$ for $(i, j)=(1,2)$ and $(2,1)$.

(4) $\partial D_{1} \cap \partial D_{2}$ is minimized up to isotopy of $D_{1}$ and $D_{2}$ in $\left(V_{i}, t_{i}\right)$.

Condition (2) implies that $\ell \cup \partial D_{i}$ does not separate the two points $H \cap K$, since $s_{i}$ connects them and is disjoint from $\ell \cup \partial D_{i}$. If such discs are found, then Theorem 1.1 follows from uniqueness of the isotopy class of meridian discs disjoint from the 
trivial arc (see Lemma 2.4 in [6]). Note that the union $\partial D_{1} \cup \partial D_{2}$ is also unique up to isotopy in $(H, K \cap H)$ if $\partial D_{1}$ and $\partial D_{2}$ intersect each other minimally. Hence, it is sufficient to find such a pair of discs $D_{1}$ and $D_{2}$, although Theorem 1.1.1 is stated for every pair of discs $D_{1}$ and $D_{2}$ such that their boundary circles intersect each other minimally. Condition (1) implies Addendum 1.2 (1).

First, we take a meridian disc $D_{i}^{\prime}$ of $V_{i}$ so that it satisfies condition (1). $D_{i}^{\prime}$ may intersect $t_{i}$ and $s_{i}$. Then we isotope $D_{i}^{\prime}$ near $\partial D_{i}^{\prime}$ along subarcs of $s_{i}$ so that it satisfies condition (2). Condition (1) is kept during this operation, because $s_{i}$ is disjoint from $\ell$. Next we will isotope $D_{i}^{\prime}$ so that it satisfies condition (3). Suppose that $\partial D_{i}^{\prime} \cup s_{j}$ has a bigon in $H-K$. Note that the bigon face $Q$ is disjoint from $\ell$ by the conditions $s_{j} \cap \ell=\emptyset$ and (1). The disc $Q$ is disjoint also from $s_{i}$, because of condition (2) and the condition that $s_{i} \cap s_{j}$ is minimal. Hence we can isotope $D_{i}^{\prime}$ near its boundary in $\left(V_{i}, t_{i}\right)$ along the disc $Q$ slightly beyond the $\operatorname{arc} Q \cap s_{j}$. This reduces the number of intersection points $\partial D_{i}^{\prime} \cap s_{j}$ by two. By repeating such operations, we can deform $D_{i}^{\prime}$ so that it satisfies condition (3). Finally, we isotope $D_{1}^{\prime}$ and $D_{2}^{\prime}$ so that they satisfy condition (4). If their boundary circles do not intersect each other minimally, then $\partial D_{1}^{\prime} \cup \partial D_{2}^{\prime}$ has a bigon $R$ in $H-K$. See 2]. For $i=1$ and $2, R$ is disjoint from $s_{i}$ by conditions (2) and (3). The circle $\ell$ intersects $R$ in subarcs. Each such subarc connects the $\operatorname{arcs} \partial D_{1}^{\prime} \cap R$ and $\partial D_{2}^{\prime} \cap R$ because of condition (1). We isotope $D_{1}^{\prime}$ near its boundary along the disc $R$ slightly beyond the arc $\partial D_{2}^{\prime} \cap R$. During the isotopy, conditions (1), (2) and (3) are kept. Repeating this process, we can deform $D_{1}^{\prime}$ and $D_{2}^{\prime}$ so that it satisfies condition (4). We isotope $t_{i}$ along $C_{i}$ to be very close to $s_{i}$ and disjoint from $D_{i}^{\prime}$. Thus we have obtained the desired pair of discs $D_{1}$ and $D_{2}$.

\section{Proof of Corollary 1.3}

We prove Corollary 1.3 in this section.

By Theorem 3 in [7, all the 1-genus 1-bridge splitting tori of a torus knot are isotopic. Hence it is enough to show that, for a certain 1-genus 1-bridge splitting, the $(p, p+2)$-torus knot has a satellite diagram of slope $(1,1)$.

Let $K$ be the $(p, p+2)$-torus knot in $S^{3}$. It is entirely contained in a standard torus $H$ which divides $S^{3}$ into two solid tori $V_{1}$ and $V_{2}$ such that $K$ goes around $p$ times longitudinally in $V_{1}$ and $p+2$ times in $V_{2}$. There is a circle $\ell$ of slope $(1,1)$ in $H$ such that $\ell$ intersects $K$ in precisely two points $x$ and $y$. Let $D_{i}$ be a meridian disc of $V_{i}$ for $i=1$ and 2 . We can take $D_{1}$ so that its boundary intersects $\ell$ only in the point $x$ and $K$ in $p$ points, one of which is $x$. We can take $D_{2}$ so that its boundary is away from $x$ and $y$ and intersects $\partial D_{1}$ in one point, $\ell$ in one point $z$, and $K$ in $p+2$ points. $x$ is the only triple intersection point of $\partial D_{1}, \partial D_{2}, K$, and $\ell$. See Figure 2, Let $s_{2}$ be a very short subarc of $K$ near $x$, and $s_{1}$ the complementary $\operatorname{arc} \operatorname{cl}\left(K-s_{2}\right)$. Because $\partial D_{2}$ is away from $x$, it is disjoint from the arc $s_{2}$. Among the $p+1$ subarcs of $s_{1}$ obtained by cutting $s_{1}$ at the $p$ points $\left(s_{1} \cap \partial D_{1}\right) \cup y$, there is an arc $\alpha$ connecting a point of $s_{1} \cap \partial D_{1}$ and a point of $\partial s_{1}$. Note that $\alpha$ is disjoint from $y$. We isotope $D_{1}$ near its boundary along the $\operatorname{arc} \alpha$ on the torus $H$. Repeating this operation, we obtain $D_{1}^{\prime}$ from $D_{1}$ such that $\partial D_{1}^{\prime}$ is disjoint from $s_{1}$. Since $s_{1}$ is disjoint from $x, \partial D_{1}^{\prime}$ intersects $\ell$ only in the single point $x$. Hence $\ell \cup \partial D_{1}^{\prime}$ does not separate the two points $H \cap K$ (though $s_{1}$ intersects $\ell$ in the point $y$ ). We call this $D_{1}^{\prime}$ simply $D_{1}$ again. Moreover, $\partial D_{2}$ intersects $\ell$ only in the single point $z$, so $\ell \cup \partial D_{2}$ does not separate the two points $H \cap K$ (though $s_{2}$ intersects 


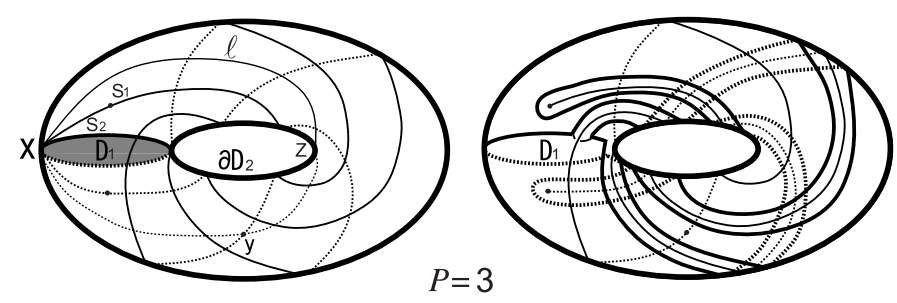

FiguRE 2.

$\ell$ in the point $x$ ). We push the interior of the arc $s_{i}$ into the interior of $V_{i}$, to form a trivial arc $t_{i}$ in $V_{i}$ for $i=1$ and 2. Note that $K$ is isotopic to $t_{1} \cup t_{2}$, and that $t_{i}$ is disjoint from $D_{i}$. Thus $H$ gives a 1-genus 1-bridge splitting of $K=t_{1} \cup t_{2}$, and $D_{1}$ and $D_{2}$ together give a Heegaard diagram of this splitting such that $\ell \cup \partial D_{i}$ does not separate the two points $H \cap K=\partial t_{i}$ for $i=1$ and 2. Theorem 1.1 with Addendum 1.2 (2) implies that $K$ has a satellite diagram of slope $\ell$.

\section{Proof of having no satellite diagram of LOngitudinal SLOpe}

In this section, we show that the torus knot $K=T(5,12)$ does not have a 1 genus 1-bridge splitting which admits a satellite diagram of longitudinal slope of one of the solid tori.

Let $\left(S^{3}, K\right)=\left(V_{1}, t_{1}\right) \cup_{H}\left(V_{2}, t_{2}\right)$ be a 1-genus 1-bridge splitting. By Theorem 3 in [7], there are cancelling discs $C_{1}, C_{2}$ of $t_{1}, t_{2}$ in $V_{1}, V_{2}$ such that $C_{1} \cap C_{2}=H \cap K$. Set $C_{i} \cap H=s_{i}$, the arc for $i=1$ and 2 . Then $L=s_{1} \cup s_{2}$ forms a simple closed curve isotopic to $K$. On one of the solid tori, say $V_{1}, L$ goes around 5 times longitudinally, and on the other solid tours $V_{2}, 12$ times longitudinally. We will show that this splitting does not admit a satellite diagram of longitudinal slope of $V_{2}$.

Let $D_{1}^{\prime}$ be a meridian disc of $V_{1}$ such that $\partial D_{1}^{\prime}$ intersects $s_{2}$ transversely in a single point $x_{0}$ and $s_{1}$ in 4 points $x_{1}, x_{2}, x_{3}, x_{4}$ which appear on $\partial D_{1}^{\prime}$ in this order. We take a meridian disc $D_{2}$ of $V_{2}$ so that:

(1) $\partial D_{2}$ is disjoint from $s_{2}$;

(2) $\partial D_{2}$ intersects $s_{1}$ transversely in 12 points $y_{1}, \ldots, y_{12}$ appearing on $\partial D_{2}$ in this order;

(3) $\partial D_{2}$ intersects $\partial D_{1}^{\prime}$ in a single point $y_{0}$ between the points $y_{12}$ and $y_{1}$ and between the points $x_{2}$ and $x_{3}$;

(4) the subarc of $L$ between $x_{0}$ and $y_{3}$ contains a point $x_{+}$of $H \cap K$; and

(5) the subarc of $L$ between $x_{0}$ and $y_{10}$ contains a point $x_{-}$of $H \cap K$.

See Figure 3, where the torus $H$ cut along $\partial D_{2}$ is described.

We form a Heegaard diagram of this splitting. We isotope $D_{1}^{\prime}$ near its boundary along subarcs of $s_{1}$ between $x_{2}$ and $x_{+}$and between $x_{4}$ and $x_{+}$. See Figure 4 , where subarcs of $\partial D_{1}^{\prime}$ in (a) are deformed to those in (b). Further, we isotope $D_{1}^{\prime}$ along subarcs of $s_{1}$ between $x_{3}$ and $x_{-}$and between $x_{1}$ and $x_{-}$similarly.

After these isotopies, $D_{1}^{\prime}$ is transformed into a disc $D_{1}$ whose boundary is disjoint from the arc $s_{1}$. We schematically describe $\partial D_{1}$ as in Figure $4(\mathrm{c})$, which implies $\partial D_{1}$ contains 2 subarcs parallel to the segment from $x_{4}$ to $x_{2}$ and 4 subarcs parallel to the segment from $x_{2}$ to $x_{+}$. We call these subarcs multiplied subarcs of $s_{1}$ in the following. 


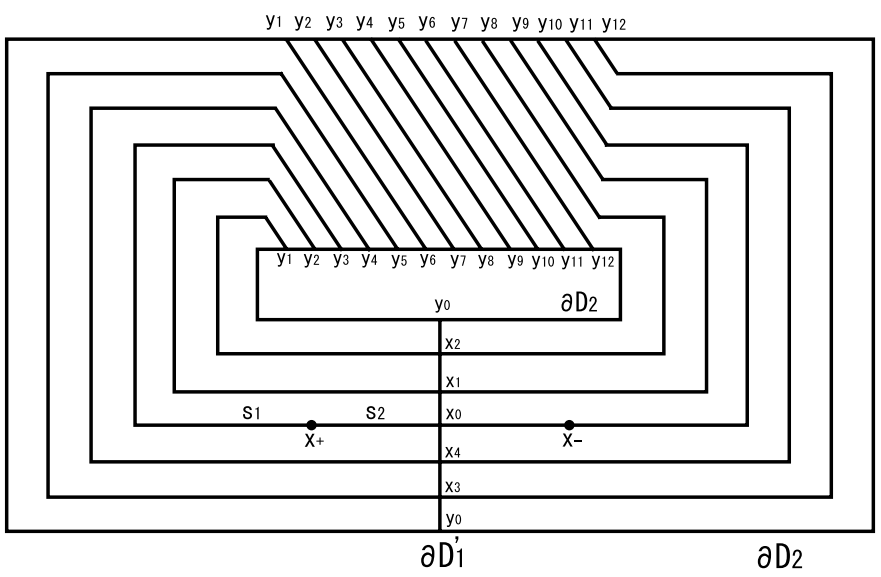

FiguRE 3.
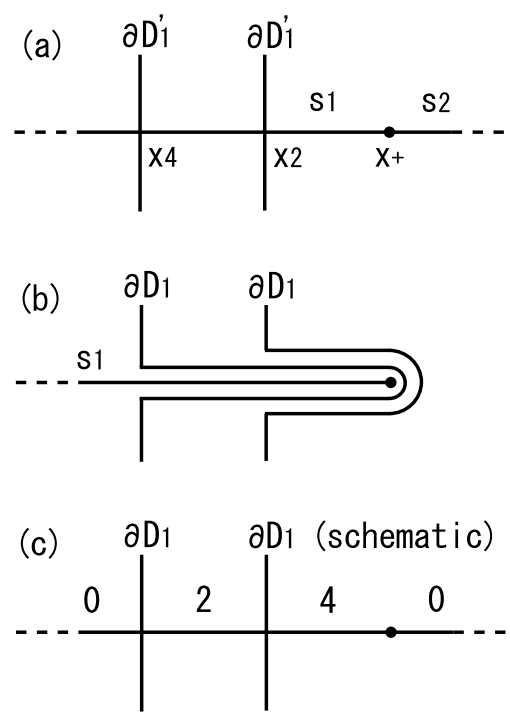

FiguRE 4.

The circles $\partial D_{1}$ and $\partial D_{2}$ together give a Heegaard diagram of the 1-genus 1bridge splitting. This diagram is minimal; that is, $\partial D_{1} \cup \partial D_{2}$ has no bigon in $H-K$. See Figure 5 .

By Theorem 1.1 and Addendum 1.2 (1), it is sufficient to confirm that there is no circle $\ell$ such that $\ell$ intersects $\partial D_{2}$ in a single point, say $z$, and $\left|\ell \cap \partial D_{1}\right|=\left|\ell \cdot \partial D_{1}\right|$. We orient the circle $\partial D_{1}$ arbitrarily. Then every multiplied subarc of $s_{1}$ contains a pair of anti-parallel subarcs of $\partial D_{1}$.

In Figure [6, the multiplied subarc of $s_{1}$ between $y_{7}$ and $y_{12}$ and that between $y_{12}$ and $y_{5}$ together separate the 2 copies of the corner of $\partial D_{2}$ between $y_{7}$ and $y_{12}$ (via $y_{8}$ ). Hence the point $z=\ell \cap \partial D_{2}$ cannot be between $y_{7}$ and $y_{12}$ (via $y_{8}$ ). (Otherwise, $\ell$ must intersect a multiplied subarc of $s_{1}$, and then intersects $\partial D_{1}$ in more than $\left|\ell \cdot \partial D_{1}\right|$ points.) Similarly, the point $z$ cannot be between $y_{12}$ and $y_{5}$ (via 


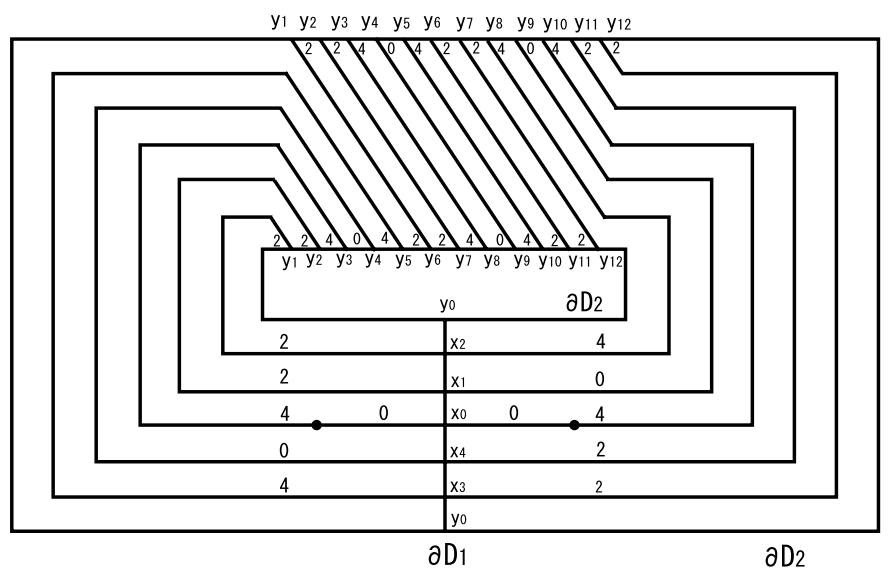

FIGURE 5.

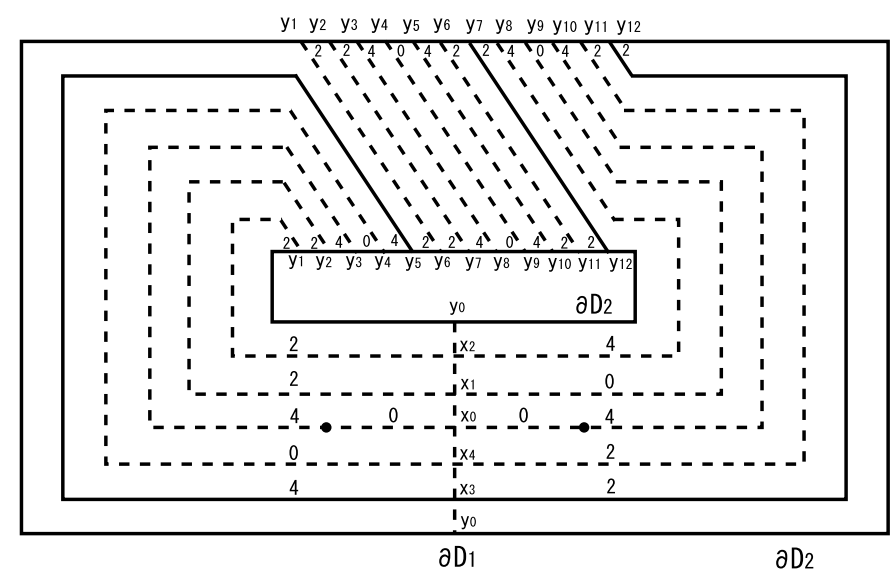

FiguRE 6.

$\left.y_{0}\right)$. Considering the multiplied subarc of $s_{1}$ between $y_{1}$ and $y_{8}$ and that between $y_{3}$ and $y_{8}$, we can see that $z$ cannot be between $y_{3}$ and $y_{8}$ (via $y_{4}$ ) nor between $y_{8}$ and $y_{1}$ (via $\left.y_{9}\right)$. Hence $z$ can be nowhere, and there is no such $\ell$.

\section{REFERENCES}

1. M. Ait Nouh and A. Yasuhara, Torus knots that cannot be untied by twisting, Rev. Mat. Complut. 14 (2001), 423-437. MR 2002j:57005

2. D.B.A. Epstein, Curves on 2-manifolds and isotopies, Acta Math. 115 (1966), 83-107. MR 35:4938

3. H. Goda, C. Hayashi and H. Song, Dehn surgeries on 2-bridge links which yield reducible 3-manifolds, preprint.

4. C. Hayashi, Genus one 1-bridge positions for the trivial knot and cabled knots, Math. Proc. Cambridge Philos. Soc. 125 (1999), no. 1, 53-65. MR 99j:57005

5. C. Hayashi, Satellite knots in 1-genus 1-bridge positions, Osaka J. Math. 36 (1999), 711-729. MR 2001a:57009]

6. C. Hayashi, 1-genus 1-bridge splittings for knots, to appear in Osaka J. Math. 41 (2004). 
7. K. Morimoto, On minimum genus Heegaard splittings of some orientable closed 3-manifolds, Tokyo J. Math. 12 (1989), 321-355. MR 91b:57020

8. K. Morimoto and M. Sakuma, On unknotting tunnels for knots, Math. Ann. 289 (1991), 143-167. MR 92e:57015

Department of Mathematics, Tokyo University of Agriculture and Technology, KoGANEI, TOKYO, 184-8588, JAPAN

E-mail address: goda@cc.tuat.ac.jp

Department of Mathematical and Physical Sciences, Faculty of Science, Japan Women's University, 2-8-1 Mejiro-dai, Bunkyo-Ku, Tokyo, 112-8681, JAPAN

E-mail address: hayashic@fc.jwu.ac.jp

Division of Mathematical Sciences, Pukyong National University, 599-1 Daeyondong, Namgu, Pusan 608-737, Korea

E-mail address: hjsong@pknu.ac.kr 